\title{
UNCONSTRAINED ASTROMETRIC ORBITS FOR HIPPARCOS STARS WITH STOCHASTIC SOLUTIONS
}

\author{
A. Goldin ${ }^{1}$ and V. V. MaKarov ${ }^{2}$ \\ Received 2006 February 1; accepted 2006 May 16
}

\begin{abstract}
A considerable number of astrometric binaries whose positions on the sky do not obey the standard model of mean position, parallax, and linear proper motion were observed by the Hipparcos satellite. Some of them remain undiscovered, and their observational data have not been properly processed with the more adequate astrometric model that includes nonlinear orbital motion. We develop an automated algorithm, based on "genetic optimization," to solve the orbital fitting problem in the most difficult setup, when no prior information about the orbital elements is available (from, e.g., spectroscopic data or radial velocity monitoring). We also offer a technique to accurately compute the probability that an orbital fit is bogus, that is, that an orbital solution is obtained for a single star, and to estimate the probability distributions for the fitting orbital parameters. We test this method on Hipparcos stars with known orbital solutions in the catalog and further apply it to 1561 stars with stochastic solutions, which may be unresolved binaries. At a confidence level of $99 \%$, orbital fits are obtained for 65 stars, most of which have not been known as binary. It is found that reliable astrometric fits can be obtained even if the period is somewhat longer than the time span of the Hipparcos mission, that is, if the orbit is not closed. A few of the new probable binaries with A-type primaries with periods 444-2015 days are chemically peculiar stars, including Ap and $\lambda$ Bootis types. The anomalous spectra of these stars are explained by admixtures of light from the unresolved, sufficiently bright and massive companions. We estimate the apparent orbits of four stars that have been identified as members of the $\approx 300 \mathrm{Myr}$ old Ursa Major kinematic group. Another four new nearby binaries may include low-mass M-type or brown dwarf companions. Follow-up spectroscopic observations in conjunction with more accurate inclination estimates will lead to better estimates of the secondary mass. Similar astrometric models and algorithms can be used for binary stars and planet hosts observed by SIM and Gaia.
\end{abstract}

Subject headings: astrometry — binaries: general

\section{INTRODUCTION}

One of the most promising and currently available ways to discover invisible companions (including brown dwarfs and giant planets) to nearby Galactic stars is to analyze high-accuracy astrometric positions of the latter for the presence of the reflex Keplerian motion caused by an orbiting companion. In the relatively near future, with the advance of such space-borne astrometric instruments as SIM PlanetQuest and Gaia, it will become one the main instruments in the search for habitable smaller planets, too. For the time being, the capabilities of the method are limited by the moderate precision of the available astrometric data. The Hipparcos Intermediate Astrometric Data (HIAD), at a singlepoint precision of roughly $10-15$ mas, are just good enough to detect brown dwarfs around nearby solar-type stars, reliably so in conjunction with spectroscopic measurements (Halbwachs et al. 2000; Pourbaix \& Jorissen 2000; Torres 2006). Perhaps giant planets of intermediate orbital period could also be detected around a few of the nearest stars.

Obtaining a robust orbital fit for an astrometric binary becomes increasingly difficult when the orbital period $P$ exceeds the time span of Hipparcos measurements, which is about 3.2 years. If the period is 6 years, still more than half of the orbit is represented in the data, and astrometric analysis may provide an independent solution. When the period is longer than 9 years, the astrometric data alone can provide only ambiguous results, since the almost linear segment of the orbit is hard to distinguish from the regular proper motion. Generally, the astrometric orbital fit is a nonlinear

\footnotetext{
1 Citadel Investment Group, 131 South Dearborn Street, Chicago, IL 60603; alexey.goldin@gmail.com.

2 Michelson Science Center, Mail Code 100-22, California Institute of Technology, 770 South Wilson Avenue, Pasadena, CA 91125; vvm@caltech.edu.
}

12-parameter adjustment problem that includes five astrometric parameters and seven orbital parameters (Appendix A). In some cases, for example, long-period orbits or highly inclined orbits aligned with the line of sight, the parameters become so entangled in the nonlinear condition equations that the fit becomes ill conditioned. Therefore, any orbital solution should be supported by analysis of confidence intervals for all fitting parameters. If the astrometric solution is sufficiently well constrained, approximate standard deviations of fitting parameters can be derived in the near vicinity of the solution point by covariance analysis, a recipe for which is given in Appendix B. Ill-conditioned solutions will have nearly singular normal matrices that cannot be inverted, or large variances for some of the parameters. In more complicated cases, some of which we investigate in this paper, the confidence intervals and probability distributions can be calculated by extensive Monte Carlo simulations. When the optimization problem is almost singular with respect to particular nonlinear parameters (most often the eccentricity), direct mapping of the objective function on a sufficiently fine grid of parameters is carried out.

Another crucial problem that arises in fitting Keplerian motion for stars previously not known as binaries is estimation of the probability of a null hypothesis, that is, that the star is single and the detected perturbation in the astrometric residuals is caused by a chance occurrence of random noise in the data or other effects unrelated to binarity. We offer a robust and straightforward (if somewhat computationally burdensome) method of confidence level estimation for a binary detection.

These techniques are tested on a set of 1561 stars in the Hipparcos Catalogue with the so-called stochastic solutions. These data often represent gross errors or failures in the data reduction. Because of the type of detector used in the main instrument on Hipparcos (a grid of long vertical slits and a photomultiplier 
behind it with a nonuniform response across the pointing field of regard), many of these failed solutions originate in a wrong assumption about the target positions, or in a lack of knowledge of the relative position and brightness of visual binary components. Successful attempts have been made to use more accurate information about stochastic stars from ground-based and Tycho-2 data sets to reprocess the published Hipparcos transit data, resulting in accurate solutions for a few hundred stars (Falin \& Mignard 1999; Fabricius \& Makarov 2000). The remaining stars with stochastic solutions are prime suspects for yet unknown binaries. Orbiting pairs with separations smaller than 0 ". 1 were not resolved by Hipparcos; on the other hand, a nonlinear apparent motion of the photocenter as small as several milliarcseconds could break the regular five-parameter solution. Long-period orbits could be detected as additional acceleration of proper motions or as discrepant proper motions from the short-term Hipparcos data and the long-term Tycho-2 definitions (Makarov \& Kaplan 2005). The overlap between such accelerating astrometric binaries and the list of stochastic objects is substantial, but presumably, many binaries on shorter orbits are left out of this analysis, as they describe a strongly nonlinear track during the 3.2-3.5 year time span of Hipparcos observations.

\section{USING HIAD TO COMPUTE ORBITS}

\subsection{Correlated Input Data}

The star abscissae data in the HIAD were derived by the two Hipparcos data reduction consortia, FAST (Fundamental Astronomy by Space Techniques) and NDAC (Norther Data Analysis Consortium), almost independently but from the same observational data (ESA 1997). Typically, each great-circle measurement produced a pair of abscissa data points, one derived by FAST and the other by NDAC. These pairs of measurements are statistically correlated. The correlation coefficient was estimated and published in the HIAD record, when appropriate. However, some of the great-circle results were processed (or accepted) by only one of the consortia, in which case the correlation is zero. Since any astrometric or orbital fit is a least-squares adjustment minimizing the $\chi^{2}$ on abscissa residuals, internal correlations must be properly taken into account.

In this paper, we obtain separate orbital fits based on NDAC and FAST data. In our judgment, these data are derived from the same observations with the same instrument, and they should be strongly correlated. The correlations given in the HIAD are probably strongly underestimated, and using a weighted combination of these data may have an adverse effect on the confidence estimation.

\subsection{Multiple Solutions and Local Minima}

The orbital motion model described in Appendix A contains three nonlinear and nine linear parameters. After $P_{0}, e$, and $T_{0}$ are fixed by a nonlinear optimization method, the rest of the parameters ( $A, B, F, G$, and five astrometric parameters) can be found by solving a linear system of equations.

Solving a multidimensional optimization problem is difficult. Deterministic methods such as the widely used Powell minimization on starting conditions can converge to different local minima. Two-dimensional optimization is sometimes marginally solvable by brute force, that is, by walking over a grid of initial values and initiating optimization from each of these starting values. For three or more dimensions, however, this approach is time-consuming, and a better approach is welcome $(\S 6)$.

\section{GENETIC OPTIMIZATION ALGORITHM}

We employ a method described by Storn \& Price (1995), which is a generalization of the genetic algorithms for optimization of continuous functions. The differential evolution algorithm is capable of handling nondifferentiable, nonlinear objective functions. It is one of the methods used in the Mathematica NMinimize routine. Although our $\chi^{2}$ objective function is differentiable everywhere except several singular points (Appendix B) and, with respect to a few parameters, linear, we have chosen this algorithm for its enhanced ability to handle complex surfaces in the parameter space with multiple minima, as discussed in $\S 4$.

In a population of potential solutions in an $n$-dimensional search space, a fixed number of vectors are randomly initialized and then evolved over time to explore the search space and to locate the minima of the objective function. At each iteration, called a generation, new difference vectors are computed by subtracting two vectors selected randomly from the current population (mutation). The difference vector is added to a third vector, which may be the current optimal vector or a randomly selected one. The resulting candidate is mixed with the current best vector, imitating the mixing of chromosomes in sexual reproduction, where each coordinate component corresponds to a chromosome. The resulting trial vector is accepted for the next generation if it yields a reduction in the value of the objective function. This process, imitating biological evolution, is not guaranteed to find the best solution that corresponds to the global minimum of the objective function, just as evolution does not always select the best possible mutation. However, the probability offinding the global minimum, or a very close solution to the global minimum, increases with the population size. Therefore, at the expense of extra computing time, we can find the "nearly" best solution within a given model, localizing most of the local minima (alternative solutions) at the same time by assuming large numbers of generation vectors. This allows us to investigate the multitude of alternative solutions rather than relying on a single possible solution that yields a sufficiently small $\chi^{2}$.

Existence of multiple solutions with roughly equal reduced $\chi^{2}$ is a warning that the pattern of noisy data is untenable for a reliable binary solution, or that some other nonbinarity effect is responsible for the observed perturbation.

\section{DISTRIBUTIONS, PROBABILITIES, AND CONFIDENCE INTERVALS}

Instead of the standard $F$-test, which is unsuitable for strongly nonlinear models, the following Monte Carlo test is used to evaluate the robustness of our orbital solutions: We generate a set of observations of a star with coordinates, parallax, and proper motions corresponding to the best-fit five-parameter model at the same observation times. Normally distributed random numbers with variances corresponding to the estimated (formal) measurement errors are added to each recorded transit time. After that, the realization is reduced for both five-parameter and 12-parameter models, resulting in $\chi_{5_{i}}^{2}$ and $\chi_{12_{i}}^{2}$ for $i=1,2, \ldots, N$, respectively. The resulting fits are compared with the original, unperturbed fits with corresponding $\chi_{12_{0}}^{2}$ and $\chi_{5_{0}}^{2}$ statistics.

We estimate the fraction of trials for which the ratio of the fiveparameter fit to that of the 12-parameter fit exceeds that ratio for the actual data:

$$
p=\frac{1}{N} \sum_{i=1}^{N} \Theta_{i}\left(\chi_{5_{i}}^{2} / \chi_{12_{i}}^{2}-\chi_{5_{0}}^{2} / \chi_{12_{0}}^{2}\right),
$$

where $\Theta(x)$ is a threshold function, equal to 1 for $x>0$, and 0 for $x<0$. The confidence of rejecting a null hypothesis is $1-p$. This test is numerically equivalent to the $F$-test for a linear problem at sufficiently high $N$. 


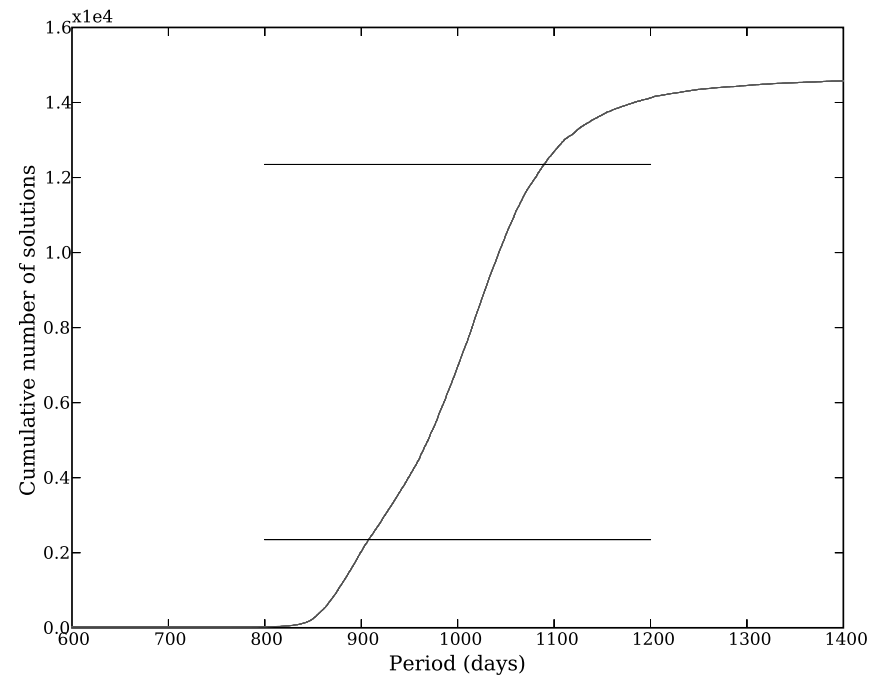

FIG. 1.-Cumulative period distribution for HIP 1366. The total number of trials is 14,700 . Horizontal lines show bounds of the $68 \%$ confidence interval.

If the probability test is passed, extensive Monte Carlo simulations are carried out to estimate the errors for each parameter. Each estimated transit time is perturbed by a normally distributed random number with a standard deviation equal to the formal transit-time error (given in the HIAD), and the reduction process is repeated. This produces a distribution of true transit times given observation, provided we have accurate transit-time error estimates and the errors are truly Gaussian. By reducing these data, we obtain the distribution of underlying parameters given observation (e.g., Fig. 1). This method of parameter-error estimation is known as parametric bootstrap (Hastie et al. 2001).

At least 1000 trials are performed for each object. From the collected Monte Carlo data, we build histograms for each fit parameter, such as $e, a_{a}$, and $T_{0}$, which are subsequently used to estimate $99 \%, 95 \%$, and $68 \%$ confidence intervals. The distribution of solutions is often significantly different from a Gaussian bell curve. With increasing numbers of measurements, and decreasing relative errors of the parameters, the parameter likelihood function becomes confined to an area where linear approximation is quite adequate for error estimation (Appendix B).

Figure 2 shows $\chi^{2}$ computed for two representative stars, as functions of period $P$ in days and eccentricity $e$, while keeping $T_{0}$

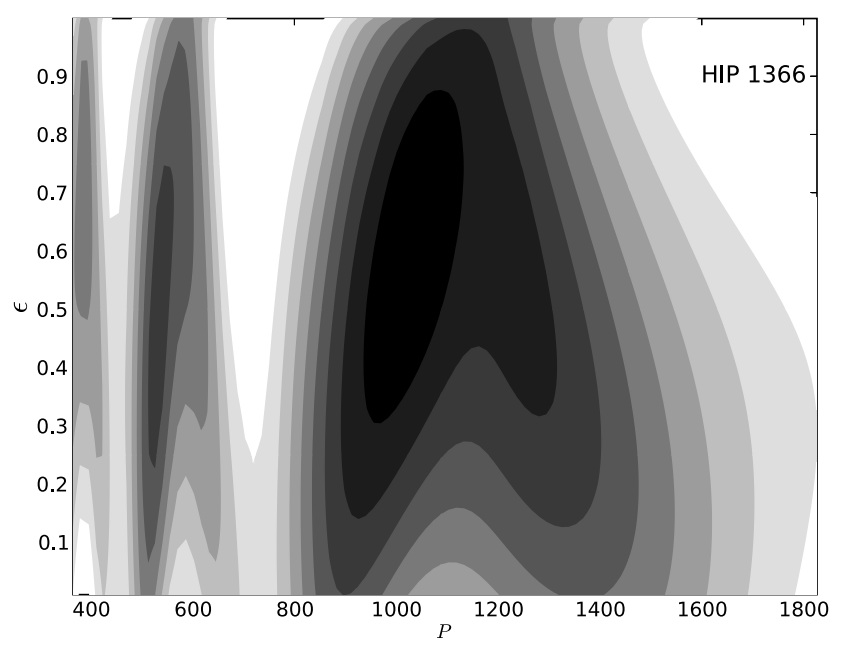

and the remaining nine parameters constant. The spacing between $\chi^{2}$ contours is 50 , the darkest color corresponding to the smallest $\chi^{2}$. We find a well-defined global minimum for HIP 1366, while for HIP 20087 we fail to find a consistent solution. Both have multiple minima (not all of them evident in this two-dimensional contour plot). Because of the nonuniform time cadence of the HIP 20087 observations and a smaller number of measurements ( 26 vs. 32 for HIP 1366), the $\chi^{2}$ map for this star is more complex. Another feature that emerges from these plots is that the $\chi^{2}$ minimization favors high-eccentricity solutions. This is not a feature of this minimization algorithm, because any $\chi^{2}$-based global optimization algorithm is biased toward high eccentricity in poorly conditioned problems. As noted by D. Pourbaix (2005), ${ }^{3}$ the effective number of degrees of freedom goes up at high eccentricity, making it possible to find a fit with anomalously small residuals if the number of observations is small. Possible solutions to this problem would be to introduce a penalty function for higheccentricity solutions or to use a Bayesian approach with a low prior probability of high-eccentricity solutions. We decided against using these techniques in this work, to keep the algorithm as simple as possible. Existing Hipparcos data do not preclude higheccentricity solutions, and rigorous estimation of prior eccentricity distributions is a project beyond the scope of this paper.

\section{COMPARISON WITH KNOWN BINARY SOLUTIONS}

To verify the algorithm, we ran it on a data set of 235 stars with known orbital solutions in the Hipparcos Catalogue. The initial period, eccentricity, and periastron time for these Hipparcos solutions were often adopted from spectroscopic data. In our work, we assume no prior knowledge of any orbital element and compute the fits from scratch. The genetic algorithm is initialized with 30 points randomly distributed in phase space, and differential evolution simulations are carried out for 100 generations for each star. The process is repeated 1000 times for each star with input data modified by adding normally distributed random numbers with standard deviations equal to the formal errors. The results of this verification analysis are shown in Figure 3, where the period of orbiting binaries derived by us and their $95 \%$ confidence intervals are put to a comparison with the periods from the Hipparcos Catalogue.

\footnotetext{
3 See http://wwwhip.obspm.fr/gaia/dms/texts/DMS-DP-02.pdf.
}

FIG. 2.-The $\chi^{2}$ contours for two stars as a function of period and eccentricity.

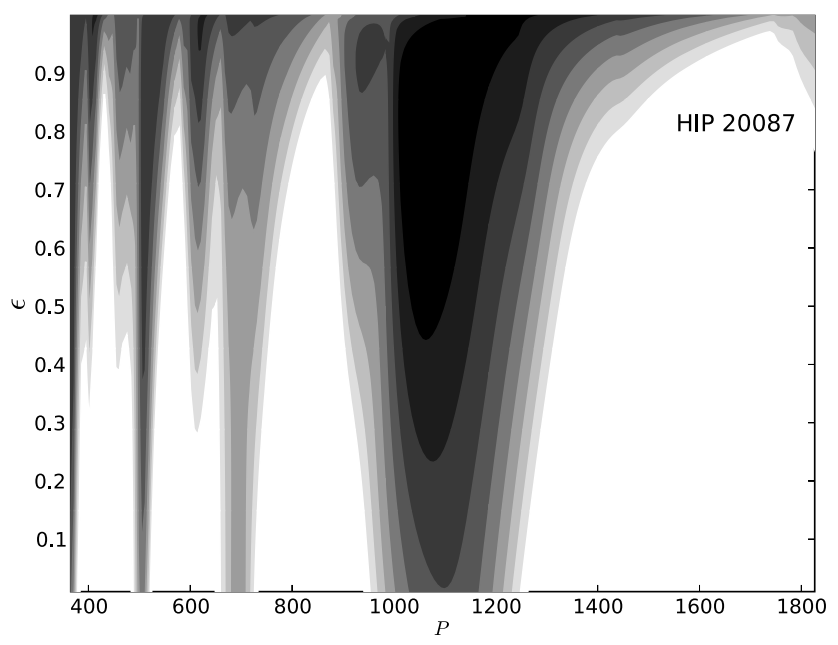




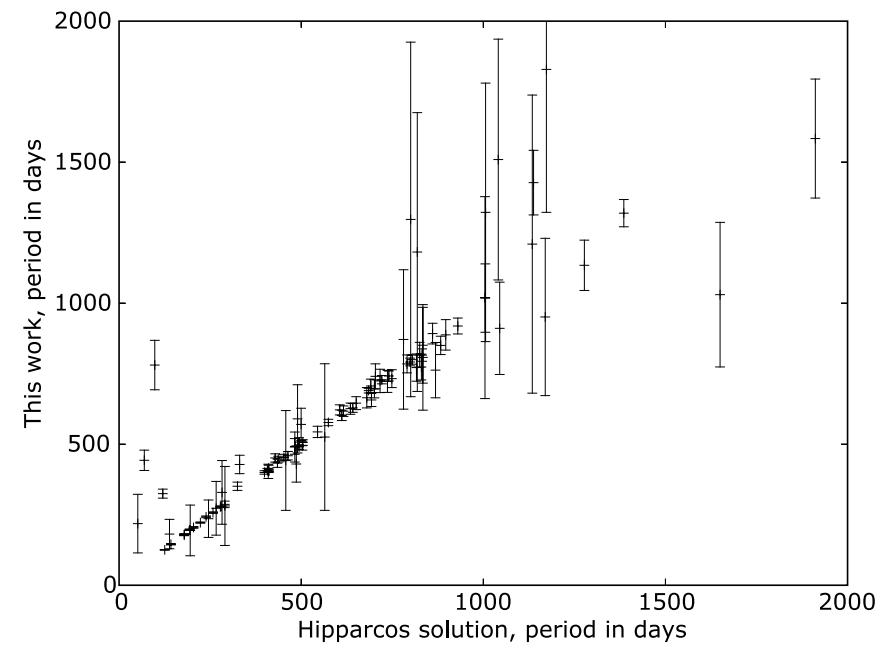

FIG. 3.- Comparison of orbital periods estimated by the genetic evolution algorithm vs. periods in the Hipparcos catalog of orbital solutions. Only FAST consortium data are shown.

The genetic algorithm fails to converge for four long-period stars, HIP 32349, 5336, 37279, and 95501. The majority of the orbital solutions are in good agreement as far as the crucial parameter $P$ is concerned. It is to a large degree an external check for our method, since the Hipparcos orbital solutions are largely based on more accurate spectroscopic orbits for the parameters in common (most notably, for $P$ and $e$ ). Recall that no a priori information or constraints are used in our algorithm. With a few exceptions, the periods shorter than 1000 days appear to be reliable. Binaries with longer periods exceeding the observation time span (typically, $3.2 \mathrm{yr}$ ) obtain uncertain solutions. Such systems will benefit from the use of spectroscopic or interferometric constraints. Four binaries with short periods in the Hipparcos Catalogue $(P<100$ days $)$ obtain much longer periods in our unconstrained fits. These could be hierarchical multiple systems, for which radial velocity monitoring tends to pick up the short-period, high velocity amplitude signal, while the astrometric method is more sensitive to long-period signals of large angular excursions.

\section{COMPARISON WITH TRADITIONAL OPTIMIZATION METHODS}

To compare the performance of our genetic optimization algorithm with traditional optimization methods, we implement a brute-force grid-search optimization algorithm and apply it to the same set of 235 stars with known orbital fits in the Hipparcos Catalogue. The minimal value of $\chi^{2}$ is obtained by Powell optimization with initial starting points for eccentricity at 0 and 0.5 , 18 starting points for period spaced evenly in logarithmic scale between 0.1 and $30 \mathrm{yr}$, and four starting points for $T_{0}$ spaced evenly between 0 and $P$. This number of starting points, $18 \times 4 \times 2=144$, corresponds to the lowest empirically determined density of the grid that still provides the highest possible rate of correct solutions. The Powell minimization is iterated until the relative change in $\chi^{2}$ is less than 0.01 . The correct solutions are defined as solutions for which the fitted period is within a factor of 1.5 of the spectroscopically derived period. For the FAST consortium data, the grid optimization routine yields 144 correct solutions, requiring on average 19,500 function estimations.

The differential evolution (DE) optimization algorithm with 30 initial points and 30 generations produces 152 correct solutions, with 140 solutions identical to the grid algorithm solutions. Each DE solution is followed by a Powell optimization on the remain- ing linear parameters with the same 0.01 tolerance on $\chi^{2}$, totaling an average of 50 function estimates.

In general, the DE global optimization algorithm requires approximately $5 \%$ as many function estimations to find the global optimum. Since the search space is only three-dimensional, the advantage of the DE algorithm is not overwhelming. This algorithm, however, allows us to use CPU-intensive methods to estimate the errors of the free parameters, which is an important and subtle aspect of orbit optimization. It requires days of CPU time instead of months on one author's laptop. For problems with a larger number of dimensions, the DE algorithm may be even more attractive. It should be noted that the rather low density of the starting parameter grid in the grid optimization technique stems from the moderate intrinsic accuracy of Hipparcos data (a few milliarcseconds), which is comparable to the detectable orbit size. Future space astrometry missions (SIM and Gaia) will operate at a factor of $10^{3}$ higher accuracy, dramatically increasing the density of the initial grid.

\section{UNCONSTRAINED SOLUTIONS FOR STOCHASTIC STARS}

We further indiscriminately applied the genetic optimization algorithm to all Hipparcos stars with stochastic solutions. Among the solutions that demonstrated stable convergence, we selected 65 stars with confidence above $99 \%$, estimated as described in $\S 4$. A literature search for these 65 stars resulted in 11 systems with sufficiently accurate spectroscopic orbits, listed in Table 1. Astrometrically estimated periods are usually quite close to the more accurate spectroscopic periods at $P<1000$ days. A few discrepant cases are present when the periods from FAST and NDAC data are different by a significant fraction of the true quantity. Generally, the FAST solutions seem to be more reliable. In all likelihood, the FAST consortium used stricter criteria in treating statistical outliers, occasional observations deviating far from the true astrometric abscissa, than the NDAC consortium did. Such outliers can disturb the nonlinear optimization process, generating multiple wrinkles and gradients in the $\chi^{2}$ function. But some of the discrepant cases find more interesting astrophysical explanations.

The star HIP 84949 (=V819 Her) is a complex triple system. The inner binary is eclipsing, of Algol type, with a period of 2.23 days. The outer G8 IV-III companion is an active spotty star rotating with a period of about 86 days ( Van Hamme et al. 1994). It can hardly be a coincidence that our best NDAC solution produced a period of 89 days. In a binary system with a variable component, the photocenter moves along the line connecting the components synchronously with the light curve, an effect known as variability-imposed motion (VIM). The magnitude of the VIM astrometric excursion depends on the angular separation, magnitude difference, and amplitude of variability. Accidentally, the VIM effect in HIP 84949 generated an orbital fit with the smallest reduced $\chi^{2}$ with NDAC data, which was not at all a typical draw, since all the percentiles correspond to long-period fits, that is, the real outer orbit. The FAST solutions are not affected by the optical variability. This case exemplifies the difficulties and hazards of purely astrometric orbital solutions for complicated multiple systems with variable components.

Another piece of evidence that most of our discoveries are real long-period binaries is the high rate of occurrence of accelerating stars (Makarov \& Kaplan 2005). This is not an independent check, though, because apparent accelerations are perceived either from the same Hipparcos observations or from a comparison of Hipparcos and Tycho-2 proper motions. We find 19 stars in common with the two catalogs of accelerating stars in Makarov \& Kaplan (2005). 
TABLE 1

Comparison of Astrometric Fits with Known Spectroscopic Orbits

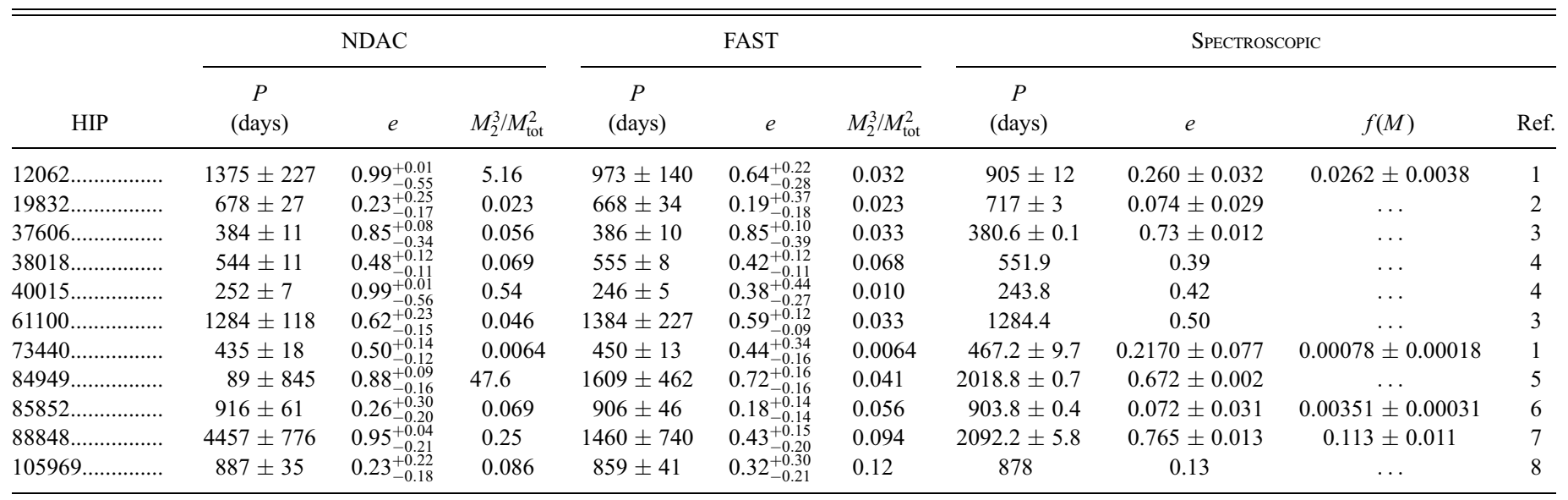

References.-(1) Latham et al. 2002; (2) Halbwachs et al. 2000; (3) Setiawan et al. 2004; (4) Halbwachs et al. 2003; (5) Scarfe et al. 1994; (6) Fekel et al. 1993; (7) Fekel et al. 2005; (8) Pourbaix \& Jorissen 2000.

Finally, the extensive Geneva-Copenhagen (GC) spectroscopic survey (Nordström et al. 2004) and other radial velocity (RV) observations in the literature provide a truly external verification of the binary nature of some of our solutions. The GC survey only includes Hipparcos stars of F, G, and K type. In some cases, only one RV measurement is available, so that orbital motion is impossible to establish. In most cases, only a few observations are available, which is not enough to derive an orbital fit but is sufficient to detect orbital motion. Combining these data with Hipparcos astrometric data might be a subject for future work. Two indicators in the GC catalog are especially useful in this respect: the probability of constant RV and a flag for spectroscopic binaries. Among the 54 new binaries from our analysis, 24 have these indicators of variable RV in the GC catalog. A few more stars were known to be spectroscopic binaries from earlier investigations, but without reliable orbit estimations. Such is the case with

HIP $23221=63$ Eri, which is orbited by a white dwarf companion;

HIP $25732=$ HD 3615 (Grenier et al. 1999);

HIP 38146 = HD 63660, a G0 III star (de Medeiros \& Mayor 1999);

HIP $43352=\mathrm{HD} 75605$, an Ursa Major member (de

Medeiros et al 2002);

HIP $62512=$ HD 111456, another Ursa Major member

(Freire Ferrero et al. 2004); and

HIP $76006=$ HD 138525, an F6 giant (de Medeiros et al 2002).

\section{OVERVIEW OF ASTROMETRIC BINARIES}

The 54 previously unknown binary systems (Tables 2-3) range in spectral type from A2 to M0. Some of the early-type stars stand out by virtue of their chemical peculiarities, most notably the $\lambda$ Bootis type star HIP 32607 ( $=\alpha$ Pic), the Am star HIP $30342(=\nu$ Pic), and the Ap star HIP 1366 (=HD 1280).

HIP $1366=H D 1280$. - This star may also be quite young. Nonmagnetic Ap stars are often found in double-lined spectroscopic binary (SB2) systems, and their companions are usually magnetic Am stars. This makes the subtle discrimination of Ap and Am stars complicated for unresolved systems. Carrier et al. (2002) derived spectroscopic orbits for $16 \mathrm{Ap}$ stars, four of which may be Am stars. They found orbital periods from 1.6 to 2422 days, which makes our solution, 1017 days, a rather common case. Generally, Carrier et al. find distributions of orbital elements similar to those of field main-sequence stars, except for a distinct deficit of systems with $P<3$ days. The latter fact is explained in terms of tidal synchronization in tight binaries, which helps to retain high rotational velocities, preventing the occurrence of chemical peculiarity. From our solution based on FAST data, the visible semimajor axis of the photocenter is about $1 \mathrm{AU}$, implying a rather massive companion, perhaps also an A star. HD 1280 has not been identified as a spectroscopic binary.

HIP $30342=H D$ 45229. - Also known as $\nu$ Pic, this Am and chemically peculiar star has drawn considerable attention in the literature. Although Am stars are often found in SB2 systems (Abt \& Levy 1985), ours is the first indication of the binary nature of this object. Using our FAST parameters for $a, \pi$, and $P$, we estimate the mass of the companion at only about onequarter of the primary mass. The FAST corrected mass function is $\left(M_{2}^{3} / M_{\mathrm{tot}}^{2}\right)_{\mathrm{FAST}}=0.09$.

HIP $32607=H D$ 50241. - Alias $\alpha$ Pic, this well-studied, bright $\lambda$ Bootis type star, was not previously known to be binary. Our derived $a$ is close to $1 \mathrm{AU}$, indicating a large mass ratio. This comports with the suggestion of Faraggiana et al. (2004) and Faraggiana $\&$ Bonifacio (2005) that many (maybe all) $\lambda$ Bootis stars are unresolved binaries with composite spectra, which would explain the observed chemical underabundances. The testable prediction here is that the unresolved companions have similar brightness to the primaries, lest their contribution to the observed spectrum be too small to account for the peculiarities of the spectral lines. An alternative hypothesis for the $\lambda$ Bootis phenomenon is based on the gas-dust separation in stellar envelopes (e.g., Andrievsky \& Paunzen 2000).

A few primaries in our sample are nearby late-type stars, whose companions can be low-mass dwarfs from the bottom of the main sequence. A large number of $\mathrm{M}$ dwarfs and substellar objects ( $\mathrm{L}$ and $\mathrm{T}$ type) are expected to roam the solar vicinity, but relatively few have been identified. The nearest low-mass stars, because of their intrinsic dimness, are traditionally important objects of investigation. Substellar companions are of particular interest, since the rate of such "failed stars" in spectroscopic binaries is currently considered to be too low in comparison with the expectation from the theoretical mass function (the "brown dwarf desert" problem).

HIP 38910.- Our solutions for $a$ and $P$ from FAST and NDAC are discrepant for this $\mathrm{K} 5 \mathrm{~V}$ star, probably because of the 
TABLE 2

Orbital Solutions from NDAC Data

\begin{tabular}{|c|c|c|c|c|c|c|c|c|}
\hline HIP & $\begin{array}{c}a_{0} \\
\text { (mas) }\end{array}$ & $\begin{array}{c}P \\
\text { (days) }\end{array}$ & $\epsilon$ & $\begin{array}{c}T_{0} \\
\text { (days) }\end{array}$ & $\begin{array}{c}\Omega \\
(\mathrm{deg})\end{array}$ & $\begin{array}{c}\omega \\
(\mathrm{deg})\end{array}$ & $\begin{array}{c}i \\
(\operatorname{deg})\end{array}$ & $\begin{array}{c}\text { Parallax } \\
\text { (mas) }\end{array}$ \\
\hline $1366 \ldots \ldots \ldots$ & $25_{-13}^{+32}$ & $1033_{-77}^{+91}$ & $0.95_{-0.40}^{+0.04}$ & $238_{-143}^{+343}$ & $263_{-156}^{+50}$ & $89_{-29}^{+175}$ & $69_{-22}^{+8}$ & $14_{-1}^{+1}$ \\
\hline $3750 \ldots \ldots \ldots$ & $18_{-3}^{+10}$ & $1646_{-321}^{+609}$ & $0.74_{-0.18}^{+0.40}$ & $704_{-569}^{+670}$ & $80_{-9}^{+6}$ & $35_{-14}^{+33}$ & $77_{-10}^{+6}$ & $12_{-1}^{+1}$ \\
\hline $3865 \ldots \ldots \ldots$ & $10_{-1}^{+5}$ & $1142_{-144}^{+146}$ & $0.47_{-0.25}^{+0.10}$ & $861_{-455}^{+251}$ & $86_{-48}^{+206}$ & $82_{-51}^{+76}$ & $44_{-13}^{+18}$ & $11_{-1}^{+1}$ \\
\hline $6273 \ldots \ldots \ldots$ & $46_{-10}^{-1}$ & $2656_{-805}^{-184}$ & $0.86_{-0.18}^{+0.23}$ & $1364_{-753}^{+1855}$ & $195_{-117}^{+59}$ & $105_{-67}^{+167}$ & $137_{-22}^{+13}$ & $34_{-1}^{+1}$ \\
\hline $6542 \ldots \ldots \ldots$ & $23_{-3}^{+8}$ & $1760_{-280}^{+550}$ & $0.65_{-0.22}^{+0.20}$ & $435_{-198}^{+520}$ & $138_{-13}^{+13}$ & $109_{-19}^{+28}$ & $54_{-7}^{+8}$ & $18_{-1}^{+1}$ \\
\hline $8525 \ldots \ldots \ldots$ & $19_{-5}^{+21}$ & $704_{-25}^{+31}$ & $0.90_{-0.37}^{+0.09}$ & $529_{-94}^{+88}$ & $233_{-183}^{+37}$ & $122_{-74}^{+159}$ & $72_{-13}^{+11}$ & $23_{-1}^{+1}$ \\
\hline $11925 \ldots \ldots \ldots$ & $16_{-3}^{+19}$ & $920_{-38}^{+236}$ & $0.71_{-0.18}^{+0.23}$ & $785_{-623}^{+100}$ & $344_{-341}^{+14}$ & $102_{-17}^{+162}$ & $94_{-5}^{+6}$ & $15_{-1}^{+1}$ \\
\hline $12062 \ldots \ldots \ldots$ & $34_{-22}^{+39}$ & $979_{-209}^{+254}$ & $0.97_{-0.54}^{+0.02}$ & $505_{-343}^{+234}$ & $203_{-152}^{+38}$ & $90_{-22}^{+176}$ & $78_{-18}^{+6}$ & $18_{-2}^{+2}$ \\
\hline $12225 \ldots \ldots \ldots$ & $24_{-1}^{+3}$ & $1148_{-116}^{+369}$ & $0.26_{-0.17}^{+0.25}$ & $939_{-436}^{+186}$ & $62_{-5}^{+176}$ & $88_{-36}^{+56}$ & $115_{-4}^{+4}$ & $22_{-1}^{+1}$ \\
\hline $12894 \ldots \ldots \ldots$ & $11_{-1}^{+3}$ & $\begin{array}{l}861_{-26}^{+27} \\
\end{array}$ & $0.46_{-0.18}^{+0.27}$ & $547_{-107}^{+139}$ & $284_{-170}^{+18}$ & $106_{-32}^{+166}$ & $123_{-9}^{+9}$ & $16_{-1}^{+1}$ \\
\hline $17022 \ldots \ldots \ldots$ & $14_{-3}^{+32}$ & $1091_{-74}^{+114}$ & $0.68_{-0.24}^{+0.10}$ & $336_{-172}^{+280}$ & $112_{-40}^{+163}$ & $121_{-80}^{+155}$ & $69_{-9}^{+12}$ & $21_{-2}^{+1}$ \\
\hline $19832 \ldots \ldots \ldots$ & $21_{-2}^{+4}$ & $669_{-25}^{+26}$ & $0.31_{-0.17}^{+0.25}$ & $264_{-109}^{+191}$ & $288_{-137}^{+62}$ & $66_{-40}^{+147}$ & $56_{-11}^{+11}$ & $46_{-3}^{+3}$ \\
\hline $21386 \ldots \ldots \ldots$ & $20_{-1}^{+2}$ & $1031_{-26}^{+33}$ & $0.23_{-0.12}^{+0.14}$ & $858_{-137}^{+112}$ & $30_{-9}^{+175}$ & $\begin{array}{l}-40 \\
89_{-68}^{+97}\end{array}$ & $98_{-2}^{+2}$ & $26_{-1}^{+3}$ \\
\hline $21965 \ldots \ldots .$. & $11_{-2}^{+30}$ & $710_{-35}^{+40}$ & $0.56_{-0.31}^{+0.12}$ & $515_{-148}^{+106}$ & $155_{-54}^{+41}$ & $100_{-28}^{+53}$ & $126_{-21}^{+14}$ & $16_{-1}^{+1}$ \\
\hline $23221 \ldots \ldots \ldots$ & $12_{-4}^{+21}$ & $829_{-56}^{+81}$ & $0.87_{-0.45}^{+0.12}$ & $273_{-155}^{+383}$ & $61_{-23}^{+174}$ & $\begin{array}{l}97_{-58}^{+83} \\
\end{array}$ & $97_{-9}^{+11}$ & $18_{-1}^{+1}$ \\
\hline $23776 \ldots \ldots .$. & $17_{-8}^{+29}$ & $709_{-45}^{+110}$ & $0.92_{-0.31}^{+0.43}$ & $190_{-107}^{+150}$ & $100_{-12}^{+159}$ & $91_{-15}^{+22}$ & $82_{-10}^{+6}$ & $28_{-1}^{+1}$ \\
\hline $25732 \ldots \ldots \ldots$ & $32_{-6}^{+13}$ & $2210_{-590}^{+2014}$ & $0.47_{-0.23}^{+0.23}$ & $845_{-496}^{+1892}$ & $279_{-176}^{+9}$ & $92_{-71}^{+101}$ & $61_{-6}^{+6}$ & $12_{-1}^{+1}$ \\
\hline $25918 \ldots \ldots \ldots$ & $51_{-6}^{+14}$ & $2723_{-963}^{+2584}$ & $0.59_{-0.13}^{+0.17}$ & $1410_{-949}^{+2035}$ & $117_{-8}^{+173}$ & $124_{-101}^{+48}$ & $53_{-6}^{+9}$ & $30_{-1}^{+1}$ \\
\hline $30223 \ldots \ldots \ldots$ & $17_{-9}^{+30}$ & $799_{-73}^{+70}$ & $0.92_{-0.65}^{+0.07}$ & $258_{-147}^{+280}$ & $137_{-60}^{+187}$ & $89_{-30}^{+64}$ & $66_{-22}^{+13}$ & $15_{-2}^{+2}$ \\
\hline $30342 \ldots \ldots \ldots$ & $10_{-3}^{+33}$ & $439_{-23}^{+27}$ & $0.75_{-0.40}^{+0.24}$ & $328_{-306}^{+85}$ & $163_{-116}^{+82}$ & $94_{-15}^{+71}$ & $107_{-11}^{+18}$ & $20_{-1}^{+1}$ \\
\hline $31703 \ldots \ldots .$. & $41_{-16}^{+34}$ & $2901_{-1412}^{+3399}$ & $0.84_{-0.11}^{+0.08}$ & $1588_{-1114}^{+3392}$ & $\begin{array}{l}99_{-21}^{+16} \\
\end{array}$ & $101_{-10}^{+13}$ & $132_{-9}^{+10}$ & $24_{-1}^{+1}$ \\
\hline $32607 \ldots \ldots \ldots$ & $36_{-2}^{+15}$ & $1618_{-325}^{+1407}$ & $0.39_{-0.17}^{+0.35}$ & $953_{-640}^{+707}$ & $24_{-5}^{+5}$ & $92_{-44}^{+22}$ & $118_{-3}^{+3}$ & $34_{-1}^{+1}$ \\
\hline $36399 \ldots \ldots \ldots$ & $22_{-5}^{+74}$ & $\begin{array}{l}995_{-39}^{+50} \\
\end{array}$ & $0.77_{-0.16}^{+0.22}$ & $815_{-767}^{+143}$ & $253_{-165}^{+26}$ & $125_{-31}^{+162}$ & $130_{-28}^{+13}$ & $39_{-1}^{+1}$ \\
\hline $37606 \ldots \ldots . .$. & $21_{-11}^{+30}$ & $380_{-11}^{+10}$ & $0.93_{-0.34}^{+0.10}$ & $81_{-45}^{+71}$ & $202_{-176}^{+165}$ & $84_{-26}^{+167}$ & $99_{-8}^{+18}$ & $44_{-2}^{+1}$ \\
\hline $38018 \ldots \ldots \ldots$ & $19_{-2}^{+3}$ & $543_{-11}^{+11}$ & $0.60_{-0.12}^{+0.16}$ & $452_{-40}^{+48}$ & $250_{-168}^{+16}$ & $51_{-28}^{+159}$ & $46_{-9}^{+8}$ & $32_{-1}^{+1}$ \\
\hline $38146 \ldots \ldots \ldots$ & $13_{-2}^{+3}$ & $902_{-20}^{+18}$ & $0.50_{-0.19}^{+0.28}$ & $581_{-105}^{+56}$ & $187_{-32}^{+158}$ & $123_{-109}^{+69}$ & $42_{-12}^{+11}$ & $10_{-1}^{+1}$ \\
\hline $38596 \ldots \ldots \ldots$ & $25_{-5}^{+23}$ & $2427_{-758}^{+2056}$ & $0.66_{-0.22}^{+0.19}$ & $1250_{-889}^{+1388}$ & $319_{-169}^{+18}$ & $92_{-48}^{+146}$ & $66_{-9}^{+10}$ & $27_{-2}^{+1}$ \\
\hline $38910 \ldots \ldots \ldots$ & $39_{-3}^{+9}$ & $2041_{-398}^{+1362}$ & $0.31_{-0.18}^{+0.29}$ & $1130_{-693}^{+797}$ & $199_{-166}^{+17}$ & $121_{-72}^{+89}$ & $56_{-9}^{+7}$ & $52_{-1}^{+1}$ \\
\hline $38980 \ldots \ldots \ldots$ & $18_{-6}^{+33}$ & $894_{-68}^{+196}$ & $0.86_{-0.44}^{+0.13}$ & $458_{-220}^{+207}$ & $292_{-174}^{+13}$ & $87_{-19}^{+171}$ & $80_{-8}^{+6}$ & $36_{-1}^{+1}$ \\
\hline $39681 \ldots \ldots \ldots$ & $23_{-3}^{+12}$ & $1964_{-484}^{+1730}$ & $0.58_{-0.37}^{+0.28}$ & $945_{-678}^{+1220}$ & $59_{-9}^{+12}$ & $99_{-30}^{+29}$ & $62_{-12}^{+10}$ & $16_{-2}^{+1}$ \\
\hline $39903 \ldots \ldots \ldots$ & $26_{-1}^{+1}$ & $901_{-15}^{+16}$ & $0.07_{-0.04}^{+0.06}$ & $224_{-143}^{+498}$ & $175_{-162}^{+172}$ & $80_{-56}^{+102}$ & $23_{-7}^{+6}$ & $49_{-1}^{+1}$ \\
\hline $40015 \ldots \ldots \ldots$ & $14_{-8}^{+19}$ & $247_{-9}^{+6}$ & $0.97_{-0.56}^{+0.02}$ & $178_{-163}^{+59}$ & $107_{-68}^{+184}$ & $94_{-18}^{+74}$ & $108_{-11}^{+22}$ & $33_{-1}^{+1}$ \\
\hline $42916 \ldots \ldots \ldots$ & $23_{-2}^{+4}$ & $817_{-17}^{+13}$ & $0.68_{-0.08}^{+0.12}$ & $338_{-61}^{+49}$ & $154_{-96}^{+66}$ & $121_{-70}^{+58}$ & $148_{-11}^{+10}$ & $37_{-1}^{+1}$ \\
\hline $43352 \ldots \ldots \ldots$ & $12_{-3}^{+37}$ & $1180_{-143}^{+253}$ & $0.74_{-0.20}^{+0.08}$ & $423_{-283}^{+515}$ & $195_{-172}^{+14}$ & $93_{-11}^{+176}$ & $119_{-21}^{+12}$ & $14_{-1}^{+1}$ \\
\hline $49546 \ldots \ldots \ldots$ & $12_{-3}^{+21}$ & $584_{-26}^{+81}$ & $0.73_{-0.32}^{+0.25}$ & $436_{-360}^{+107}$ & $326_{-176}^{+9}$ & $82_{-22}^{+170}$ & $92_{-5}^{+6}$ & $15_{-1}^{+1}$ \\
\hline $50180 \ldots \ldots \ldots$ & $35_{-4}^{+19}$ & $1764_{-327}^{+1496}$ & $0.32_{-0.18}^{+0.26}$ & $978_{-643}^{+789}$ & $192_{-178}^{+6}$ & $150_{-90}^{+132}$ & $79_{-6}^{+4}$ & $29_{-1}^{+1}$ \\
\hline $54424 \ldots \ldots .$. & $10_{-1}^{+2}$ & $1177_{-165}^{+172}$ & $0.50_{-0.17}^{+0.19}$ & $654_{-296}^{+338}$ & $278_{-169}^{+42}$ & $95_{-42}^{+149}$ & $135_{-11}^{+12}$ & $13_{-1}^{+1}$ \\
\hline $54746 \ldots \ldots \ldots$ & $14_{-3}^{+23}$ & $975_{-44}^{+58}$ & $0.88_{-0.18}^{+0.11}$ & $129_{-86}^{+198}$ & $79_{-13}^{+24}$ & $124_{-25}^{+33}$ & $68_{-10}^{+10}$ & $19_{-1}^{+1}$ \\
\hline $61100 \ldots \ldots .$. & $35_{-5}^{+26}$ & $1270_{-92}^{+145}$ & $0.65_{-0.14}^{+0.24}$ & $137_{-96}^{+917}$ & $175_{-10}^{+7}$ & $76_{-15}^{+14}$ & $63_{-6}^{+8}$ & $41_{-1}^{+1}$ \\
\hline $62512 \ldots \ldots$. & $44_{-2}^{+5}$ & $1510_{-133}^{+456}$ & $0.16_{-0.09}^{+0.25}$ & $244_{-143}^{+638}$ & $31_{-3}^{+177}$ & $154_{-126}^{+24}$ & $106_{-2}^{+2}$ & $40_{-1}^{+1}$ \\
\hline $73440 \ldots \ldots \ldots$ & $13_{-7}^{+22}$ & $436_{-18}^{+14}$ & $0.94_{-0.48}^{+0.05}$ & $85_{-60}^{+299}$ & $229_{-144}^{+43}$ & $88_{-37}^{+147}$ & $70_{-17}^{+13}$ & $30_{-1}^{+1}$ \\
\hline $75401 \ldots \ldots .$. & $22_{-4}^{+12}$ & $1171_{-233}^{+630}$ & $0.62_{-0.21}^{+0.24}$ & $753_{-503}^{+410}$ & $283_{-170}^{+14}$ & $101_{-56}^{+127}$ & $68_{-9}^{+8}$ & $19_{-1}^{+1}$ \\
\hline $76006 \ldots \ldots . .$. & $10_{-4}^{+24}$ & $584_{-31}^{+27}$ & $0.86_{-0.36}^{+0.13}$ & $342_{-99}^{+97}$ & $\begin{array}{l}95_{-25}^{+54} \\
\end{array}$ & $97_{-12}^{+43}$ & $64_{-19}^{+16}$ & $15_{-1}^{+1}$ \\
\hline $78970 \ldots \ldots \ldots$ & $12_{-1}^{+3}$ & $868_{-54}^{+54}$ & $0.28_{-0.15}^{+0.28}$ & $398_{-129}^{+265}$ & $197_{-173}^{+13}$ & $91_{-40}^{+149}$ & $67_{-7}^{+7}$ & $22_{-1}^{+1}$ \\
\hline $80884 \ldots \ldots \ldots$ & $14_{-1}^{+2}$ & $992_{-41}^{+93}$ & $0.49_{-0.16}^{+0.19}$ & $321_{-288}^{+620}$ & $292_{-176}^{+8}$ & $109_{-21}^{+167}$ & $70_{-7}^{+6}$ & $12_{-1}^{+1}$ \\
\hline $84062 \ldots \ldots$. & $13_{-2}^{+28}$ & $746_{-42}^{+41}$ & $0.68_{-0.31}^{+0.10}+0$ & $108_{-82}^{+568}$ & $\begin{array}{l}170_{-151}^{+108} \\
\end{array}$ & $94_{-78}^{+155}$ & $53_{-13}^{+17}$ & $35_{-1}^{+1}$ \\
\hline $84223 \ldots \ldots .$. & $41_{-12}^{+87}$ & $4219_{-1992}^{+5366}$ & $0.76_{-0.35}^{+0.31}$ & $2705_{-1840}^{+5357}$ & $169_{-165}^{+184}$ & $105_{-22}^{+59}$ & $102_{-7}^{+8}$ & $46_{-2}^{+2}$ \\
\hline $84949 \ldots \ldots \ldots$ & $28_{-10}^{+37}$ & $2079_{-645}^{+1136}$ & $0.91_{-0.16}^{+0.08}$ & $808_{-535}^{+1006}$ & $127_{-17}^{+16}$ & $75_{-16}^{+11}$ & $71_{-8}^{+10}$ & $15_{-1}^{+1}$ \\
\hline $85852 \ldots \ldots \ldots$ & $9_{-1}^{+2}$ & $944_{-51}^{+69}$ & $0.36_{-0.19}^{+0.30}$ & $653_{-375}^{+197}$ & $211_{-67}^{+134}$ & $92_{-60}^{+134}$ & $125_{-10}^{+12}$ & $11_{-1}^{+1}$ \\
\hline $88848 \ldots \ldots \ldots$ & $40_{-6}^{+19}$ & $1948_{-436}^{+1055}$ & $0.67_{-0.21}^{+0.19}$ & $622_{-476}^{+858}$ & $250_{-178}^{+5}$ & $105_{-16}^{+171}$ & $79_{-3}^{+3}$ & $30_{-1}^{+1}$ \\
\hline $90355 \ldots \ldots \ldots$ & $13_{-2}^{+24}$ & $297_{-9}^{+8}$ & $0.51_{-0.23}^{+0.46}$ & $161_{-40}^{+54}$ & $177_{-127}^{+46}$ & $97_{-54}^{+139}$ & $134_{-22}^{+14}$ & $30_{-2}^{+2}$ \\
\hline $93137 \ldots \ldots .$. & $13_{-2}^{+6}$ & $847_{-41}^{+66}$ & $0.59_{-0.19}^{+0.29}$ & $459_{-105}^{+177}$ & $33_{-8}^{+8}$ & $85_{-21}^{+23}$ & $80_{-7}^{+6}$ & $17_{-1}^{+1}$ \\
\hline $94347 \ldots \ldots \ldots$ & $14_{-2}^{+4}$ & $633_{-20}^{+20}$ & $0.45_{-0.16}^{+0.23}$ & $377_{-72}^{+101}$ & $35_{-21}^{+120}$ & $76_{-26}^{+30}$ & $143_{-12}^{+11}$ & $22_{-1}^{+1}$ \\
\hline $94802 \ldots \ldots \ldots$ & $18_{-1}^{+1}$ & $1069_{-35}^{+47}$ & $0.15_{-0.08}^{+0.10}$ & $571_{-206}^{+163}$ & $87_{-8}^{+10}$ & $77_{-43}^{+65}$ & $132_{-5}^{+12}$ & $23_{-1}^{+1}$ \\
\hline $97063 \ldots \ldots . .$. & $19_{-2}^{+5}$ & $1422_{-177}^{+656}$ & $0.36_{-0.21}^{+0.28}$ & $673_{-356}^{+612}$ & $201_{-139}^{+92}$ & $99_{-60}^{+147}$ & $36_{-11}^{+11}$ & $28_{-1}^{+1}$ \\
\hline $97690 \ldots \ldots . .$. & $9_{-1}^{+-2}$ & $1007_{-122}^{+108}$ & $0.44_{-0.25}^{+0.21}$ & $544_{-340}^{+300}$ & $41_{-19}^{+167}$ & $87_{-57}^{-80}$ & $63_{-12}^{+11}$ & $11_{-1}^{+1}$ \\
\hline $98375 \ldots \ldots . .$. & $12_{-5}^{+-22}$ & $285_{-15}^{+14}$ & $0.96_{-0.47}^{+0.23}$ & $135_{-94}^{+101}$ & $181_{-162}^{+27}$ & $97_{-29}^{+161}$ & $92_{-9}^{+10}$ & $21_{-1}^{+1}$ \\
\hline $103287 \ldots \ldots$ & $31_{-3}^{+15}$ & $1484_{-258}^{+602}$ & $0.50_{-0.29}^{+0.34}$ & $878_{-690}^{+478}$ & $339_{-178}^{+5}$ & $119_{-28}^{+165}$ & $86_{-7}^{+5}$ & $24_{-2}^{+2}$ \\
\hline $104440 \ldots \ldots$ & $68_{-27}^{+82}$ & $2145_{-914}^{+3036}$ & $0.70_{-0.08}^{+0.29}$ & $1073_{-716}^{+2310}$ & $13_{-4}^{+180}$ & $148_{-135}^{+32}$ & $90_{-2}^{+2}$ & $51_{-1}^{+1}$ \\
\hline $105969 \ldots \ldots$ & $13_{-1}^{+2}$ & $885_{-36}^{+34}$ & $0.34_{-0.18}^{+0.08}$ & $415_{-145}^{+179}$ & $109_{-24}^{+178}$ & $\begin{array}{l}130_{-107}^{+47} \\
\end{array}$ & $133_{-9}^{+11}$ & $15_{-1}^{+1}$ \\
\hline $107143 \ldots \ldots$ & $36_{-3}^{+9}$ & $1371_{-111}^{+562}$ & $0.28_{-0.17}^{+0.34}$ & $699_{-628}^{+653}$ & $111_{-30}^{+175}$ & $77_{-51}^{+95}$ & $46_{-9}^{+10}$ & $41_{-2}^{+2}$ \\
\hline $110291 \ldots \ldots$ & $17_{-8}^{+26}$ & $757_{-58}^{+82}$ & $0.92_{-0.34}^{+0.07}$ & $420_{-337}^{+295}$ & $72_{-27}^{+171}$ & $85_{-24}^{+86}$ & $99_{-8}^{+12}$ & $18_{-2}^{+3}$ \\
\hline $113638 \ldots \ldots$ & $23_{-15}^{+34}$ & $356_{-18}^{+11}$ & $0.91_{-0.53}^{+0.08}$ & $206_{-70}^{+113}$ & $158_{-51}^{+35}$ & $88_{-14}^{+32}$ & $76_{-24}^{+11}$ & $30_{-5}^{+3}$ \\
\hline $117622 \ldots \ldots$ & $14_{-2}^{+46}$ & $1011_{-65}^{+104}$ & $0.59_{-0.35}^{+0.40}$ & $285_{-193}^{+549}$ & $28_{-19}^{+26}$ & $99_{-31}^{+44}$ & $126_{-25}^{+15}$ & $20_{-2}^{+1}$ \\
\hline $118212 \ldots \ldots$ & $35_{-3}^{+4}$ & $885_{-17}^{+28}$ & $0.56_{-0.10}^{+0.12}$ & $553_{-63}^{+76}$ & $110_{-6}^{+6}$ & $110_{-17}^{+15}$ & $115_{-5}^{+5}$ & $67_{-3}^{+3}$ \\
\hline
\end{tabular}


TABLE 3

Orbital Solutions from FAST Data

\begin{tabular}{|c|c|c|c|c|c|c|c|c|}
\hline HIP & $\begin{array}{c}a_{0} \\
\text { (mas) }\end{array}$ & $\begin{array}{c}P \\
\text { (days) }\end{array}$ & $\epsilon$ & $\begin{array}{c}T_{0} \\
\text { (days) }\end{array}$ & $\begin{array}{c}\Omega \\
(\mathrm{deg})\end{array}$ & $\begin{array}{c}\omega \\
(\mathrm{deg})\end{array}$ & $\begin{array}{c}i \\
(\mathrm{deg})\end{array}$ & $\begin{array}{c}\text { Parallax } \\
\text { (mas) }\end{array}$ \\
\hline $1366 \ldots \ldots \ldots \ldots \ldots . .$. & $12_{-2}^{+9}$ & $1006_{-102}^{+88}$ & $0.62_{-0.25}^{+0.31}$ & $272_{-174}^{+480}$ & $294_{-171}^{+18}$ & $70_{-42}^{+149}$ & $63_{-10}^{+10}$ & $15_{-1}^{+1}$ \\
\hline $3750 \ldots \ldots \ldots \ldots \ldots \ldots$ & $17_{-3}^{+10}$ & $1640_{-403}^{+809}$ & $0.82_{-0.20}^{+0.17}$ & $779_{-575}^{+615}$ & $82_{-6}^{+7}$ & $36_{-21}^{+36}$ & $86_{-8}^{+5}$ & $13_{-1}^{+1}$ \\
\hline 3865 ..................... & $24_{-13}^{+20}$ & $1325_{-277}^{+371}$ & $0.97_{-0.28}^{+0.02}$ & $527_{-432}^{+599}$ & $54_{-42}^{+236}$ & $82_{-38}^{+10}$ & $68_{-24}^{+9}$ & $10_{-1}^{+1}$ \\
\hline $6273 \ldots \ldots \ldots \ldots \ldots \ldots$ & $55_{-15}^{+67}$ & $3261_{-1316}^{+3108}$ & $0.84_{-0.28}^{+0.20}$ & $1995_{-1236}^{+3090}$ & $135_{-37}^{+131}$ & $95_{-78}^{+80}$ & $128_{-15}^{+14}$ & $34_{-2}^{+1}$ \\
\hline $6542 \ldots \ldots \ldots \ldots \ldots \ldots$ & $22_{-3}^{+8}$ & $1807_{-282}^{+570}$ & $0.62_{-0.26}^{+0.22}$ & $441_{-224}^{+555}$ & $144_{-11}^{+10}$ & $99_{-16}^{+23}$ & $50_{-7}^{+7}$ & $16_{-1}^{+1}$ \\
\hline $8525 \ldots \ldots \ldots \ldots \ldots \ldots$ & $19_{-6}^{+27}$ & $731_{-28}^{+36}$ & $0.86_{-0.45}^{+0.13}$ & $576_{-130}^{+84}$ & $231_{-191}^{+30}$ & $105_{-70}^{+130}$ & $72_{-11}^{+8}$ & $24_{-1}^{+-1}$ \\
\hline $11925 \ldots \ldots \ldots \ldots \ldots$ & $13_{-2}^{+7}$ & $974_{-61}^{+181}$ & $0.64_{-0.20}^{+0.45}$ & $252_{-199}^{+626}$ & $350_{-347}^{+8}$ & $125_{-20}^{+161}$ & $97_{-5}^{+6}$ & $16_{-1}^{+1}$ \\
\hline 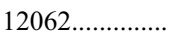 & $16_{-4}^{+50}$ & $1028_{-166}^{+123}$ & $0.78_{-0.28}^{+0.21}$ & $348_{-200}^{+335}$ & $184_{-113}^{+64}$ & $90_{-37}^{+118}$ & $51_{-17}^{+27}$ & $19_{-1}^{+1}$ \\
\hline $12225 \ldots \ldots \ldots \ldots \ldots$ & $25_{-2}^{+5}$ & $1247_{-175}^{+1148}$ & $0.31_{-0.21}^{+0.32}$ & $976_{-707}^{+421}$ & $53_{-8}^{+174}$ & $71_{-34}^{+100}$ & $121_{-4}^{+5}$ & $21_{-1}^{+1}$ \\
\hline 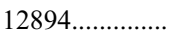 & $10_{-1}^{+\frac{3}{3}}$ & $862_{-31}^{+33}$ & $0.35_{-0.19}^{+0.36}$ & $513_{-135}^{+238}$ & $281_{-166}^{+23}$ & $85_{-49}^{+147}$ & $119_{-8}^{+8}$ & $15_{-1}^{+1}$ \\
\hline 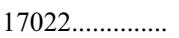 & $15_{-2}^{+8}$ & $1119_{-63}^{+98}$ & $0.63_{-0.17}^{+0.28}$ & $423_{-169}^{+234}$ & $83_{-13}^{+184}$ & $96_{-82}^{+81}$ & $65_{-6}^{+7}$ & $20_{-1}^{+1}$ \\
\hline $19832 \ldots \ldots \ldots \ldots . . . .$. & $21_{-3}^{+7}$ & $662_{-34}^{+34}$ & $0.32_{-0.18}^{+0.38}$ & $383_{-334}^{+231}$ & $170_{-136}^{+37}$ & $75_{-32}^{+95}$ & $47_{-11}^{+13}$ & $43_{-4}^{+3}$ \\
\hline 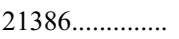 & $20_{-1}^{+1}$ & $1044_{-23}^{+29}$ & $0.23_{-0.11}^{+0.13}$ & $909_{-134}^{+89}$ & $203_{-178}^{+5}$ & $59_{-38}^{+136}$ & $100_{-2}^{+2}$ & $25_{-1}^{+1}$ \\
\hline 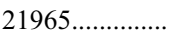 & $10_{-1}^{+2}$ & $718_{-20}^{+21}$ & $0.30_{-0.17}^{+0.27}$ & $456_{-331}^{+117}$ & $167_{-13}^{+167}$ & $104_{-41}^{+48}$ & $123_{-7}^{+8}$ & $15_{-1}^{+1}$ \\
\hline $23221 \ldots \ldots \ldots \ldots . . . . . . . .$. & $17_{-7}^{+22}$ & $862_{-53}^{+71}$ & $0.93_{-0.22}^{+0.06}$ & $257_{-102}^{+123}$ & $40_{-9}^{+11}$ & $109_{-12}^{+27}$ & $93_{-5}^{+7}$ & $19_{-1}^{+1}$ \\
\hline $23776 \ldots \ldots \ldots \ldots \ldots$ & $12_{-4}^{+26}$ & $718_{-38}^{+35}$ & $0.84_{-0.34}^{+0.15}$ & $245_{-86}^{+99}$ & $101_{-11}^{+156}$ & $96_{-23}^{+34}$ & $77_{-12}^{+9}$ & $28_{-1}^{+1}$ \\
\hline $25732 \ldots \ldots \ldots \ldots \ldots$ & $31_{-8}^{+19}$ & $2707_{-1079}^{+2803}$ & $0.50_{-0.30}^{+0.24}$ & $1341_{-964}^{+2667}$ & $113_{-10}^{+170}$ & $146_{-128}^{+31}$ & $62_{-7}^{+10}$ & $12_{-1}^{+1}$ \\
\hline $25918 \ldots \ldots \ldots \ldots \ldots$ & $60_{-10}^{+24}$ & $3109_{-1086}^{+2525}$ & $0.53_{-0.13}^{+0.19}$ & $1560_{-1084}^{+2098}$ & $123_{-12}^{+19}$ & $124_{-34}^{+42}$ & $52_{-6}^{+9}$ & $31_{-1}^{+1}$ \\
\hline 30223................. & $10_{-2}^{+30}$ & $796_{-64}^{+78}$ & $0.56_{-0.32}^{+0.43}$ & $\begin{array}{l}240_{-126}^{+261} \\
\text { - }\end{array}$ & $146_{-42}^{+170}$ & $88_{-56}^{+78}$ & $51_{-13}^{+22}$ & $14_{-2}^{+2}$ \\
\hline $30342 \ldots \ldots \ldots \ldots . . .$. & $6_{-1}^{+20}$ & $442_{-25}^{+19}$ & $0.46_{-0.27}^{+0.53}$ & $206_{-171}^{+162}$ & $112_{-72}^{+129}$ & $93_{-38}^{+75}$ & $119_{-17}^{+17}$ & $20_{-1}^{+1}$ \\
\hline 31703................. & $36_{-13}^{+34}$ & $2676_{-1233}^{+3382}$ & $0.83_{-0.11}^{+0.09}$ & $1382_{-981}^{+3371}$ & $102_{-50}^{+27}$ & $106_{-26}^{+25}$ & $139_{-10}^{+11}$ & $25_{-1}^{+1}$ \\
\hline $32607 \ldots \ldots \ldots \ldots . . .$. & $35_{-2}^{+11}$ & $1528_{-264}^{+1013}$ & $0.37_{-0.16}^{+0.33}$ & $915_{-613}^{+615}$ & $20_{-5}^{+4}$ & $89_{-43}^{+23}$ & $121_{-3}^{+3}$ & $34_{-1}^{+1}$ \\
\hline 36399................ & $20_{-3}^{+12}$ & $1008_{-30}^{+32}$ & $0.69_{-0.10}^{+0.21}$ & $101_{-58}^{+541}$ & $202_{-115}^{+55}$ & $113_{-46}^{+166}$ & $143_{-20}^{+12}$ & $39_{-1}^{+1}$ \\
\hline $37606 \ldots \ldots \ldots \ldots \ldots$ & $18_{-8}^{+34}$ & $383_{-9}^{+9}$ & $0.89_{-0.38}^{+0.10}$ & $97_{-40}^{+45}$ & $201_{-176}^{+10}$ & $87_{-11}^{+176}$ & $96_{-6}^{+12}$ & $43_{-2}^{+2}$ \\
\hline $38018 \ldots \ldots \ldots \ldots . . . . .$. & $18_{-1}^{+1}$ & $556_{-9}^{+8}$ & $0.44_{-0.11}^{+0.10}$ & $509_{-460}^{+34}$ & $265_{-175}^{+9}$ & $41_{-15}^{+172}$ & $48_{-7}^{+6}$ & $33_{-1}^{+1}$ \\
\hline $38146 \ldots \ldots \ldots \ldots \ldots$ & $12_{-1}^{+\frac{1}{2}}$ & $889_{-26}^{+22}$ & $0.57_{-0.13}^{+0.16}$ & $\begin{array}{l}522_{-88}^{+400} \\
\text { +69 }\end{array}$ & $190_{-169}^{+26}$ & $151_{-50}^{+153}$ & $44_{-11}^{+10}$ & $11_{-1}^{+1}$ \\
\hline $38596 \ldots \ldots \ldots \ldots \ldots . . . . .$. & $27_{-9}^{+35}$ & $2566_{-918}^{+2582}$ & $0.68_{-0.20}^{+0.27}$ & $1298_{-903}^{+1905}$ & $288_{-170}^{+18}$ & $99_{-41}^{+157}$ & $62_{-13}^{+18}$ & $31_{-1}^{+1}$ \\
\hline 38910................. & $72_{-29}^{+84}$ & $3437_{-1354}^{+3199}$ & $0.32_{-0.22}^{+0.50}$ & $2063_{-1480}^{+3008}$ & $164_{-109}^{+169}$ & $117_{-81}^{+60}$ & $53_{-14}^{+21}$ & $51_{-1}^{+2}$ \\
\hline $38980 \ldots \ldots \ldots \ldots \ldots$ & $15_{-4}^{+26}$ & $991_{-136}^{+111}$ & $0.74_{-0.37}^{+0.25}$ & $439_{-300}^{+247}$ & $295_{-176}^{+11}$ & $105_{-32}^{+164}$ & $76_{-8}^{+8}$ & $34_{-1}^{+1}$ \\
\hline 39681................ & $21_{-3}^{+11}$ & $1579_{-225}^{+534}$ & $0.66_{-0.21}^{+0.21}$ & $1031_{-813}^{+468}$ & $60_{-8}^{+10}$ & $72_{-20}^{+31}$ & $72_{-6}^{+8}$ & $15_{-2}^{+-1}$ \\
\hline 39903 ................ & $30_{-1}^{+1}$ & $922_{-11}^{+11}$ & $0.14_{-0.04}^{+0.05}$ & $125_{-53}^{+51}$ & $339_{-334}^{+16}$ & $35_{-21}^{+162}$ & $32_{-4}^{+4}$ & $49_{-1}^{+1}$ \\
\hline $40015 \ldots \ldots \ldots \ldots \ldots$ & $7_{-1}^{+18}$ & $245_{-6}^{+5}$ & $0.56_{-0.27}^{+0.43}$ & $196_{-70}^{+32}$ & $107_{-33}^{+177}$ & $116_{-45}^{+51}$ & $112_{-12}^{+15}$ & $33_{-1}^{+1}$ \\
\hline 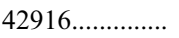 & $23_{-2}^{+3}$ & $833_{-11}^{+15}$ & $0.69_{-0.08}^{+0.08}$ & $384_{-31}^{+36}$ & $142_{-77}^{+84}$ & $104_{-75}^{+71}$ & $150_{-10}^{+9}$ & $36_{-1}^{+1}$ \\
\hline $43352 \ldots \ldots \ldots \ldots \ldots$ & $9_{-1}^{+2}$ & $1174_{-113}^{+187}$ & $0.51_{-0.14}^{+0.19}$ & $451_{-264}^{+488}$ & $208_{-161}^{+25}$ & $125_{-44}^{+156}$ & $138_{-9}^{+11}$ & $13_{-1}^{+1}$ \\
\hline $49546 \ldots \ldots \ldots \ldots \ldots . . .$. & $11_{-3}^{+18}$ & $566_{-23}^{+34}$ & $0.78_{-0.25}^{+0.21}$ & $466_{-104}^{+72}$ & $328_{-174}^{+11}$ & $79_{-26}^{+165}$ & $84_{-8}^{+5}$ & $16_{-1}^{+1}$ \\
\hline $50180 \ldots \ldots \ldots \ldots . . . . . .$. & $35_{-4}^{+18}$ & $1838_{-410}^{+1754}$ & $0.37_{-0.18}^{+0.31}$ & $1041_{-680}^{+820}$ & $194_{-179}^{+5}$ & $159_{-101}^{+150}$ & $77_{-5}^{+4}$ & $30_{-1}^{+1}$ \\
\hline $54424 \ldots \ldots \ldots \ldots \ldots$ & $10_{-1}^{+2}$ & $1124_{-211}^{+270}$ & $0.55_{-0.17}^{+0.18}$ & $615_{-300}^{+438}$ & $300_{-159}^{+27}$ & $126_{-91}^{+165}$ & $128_{-10}^{+11}$ & $15_{-1}^{+1}$ \\
\hline $54746 \ldots \ldots \ldots \ldots . . . . .$. & $12_{-2}^{+17}$ & $1018_{-50}^{+79}$ & $0.83_{-0.16}^{+0.16}$ & $166_{-86}^{+196}$ & $85_{-11}^{+16}$ & $127_{-25}^{+24}$ & $70_{-9}^{+8}$ & $19_{-1}^{+1}$ \\
\hline 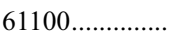 & $31_{-3}^{+5}$ & $1420_{-157}^{+303}$ & $0.61_{-0.10}^{+0.12}$ & $201_{-135}^{+817}$ & $176_{-14}^{+6}$ & $51_{-22}^{+32}$ & $57_{-6}^{+6}$ & $41_{-1}^{+1}$ \\
\hline $62512 \ldots \ldots \ldots \ldots \ldots$ & $45_{-3}^{+10}$ & $1600_{-190}^{+703}$ & $0.20_{-0.13}^{+0.27}$ & $299_{-217}^{+1066}$ & $31_{-2}^{+176}$ & $164_{-92}^{+10}$ & $108_{-2}^{+0}$ & $39_{-1}^{+1}$ \\
\hline $73440 \ldots \ldots \ldots \ldots . . . . . .$. & $8_{-2}^{+17}$ & $448_{-13}^{+13}$ & $0.65_{-0.26}^{+0.34}$ & $109_{-65}^{+66}$ & $244_{-163}^{+24}$ & $84_{-37}^{+156}$ & $68_{-10}^{+11}$ & $30_{-1}^{+1}$ \\
\hline $75401 \ldots \ldots \ldots \ldots \ldots$ & $19_{-3}^{+12}$ & $963_{-89}^{+707}$ & $0.47_{-0.29}^{+0.39}$ & $677_{-347}^{+292}$ & $121_{-11}^{+177}$ & $132_{-54}^{+39}$ & $74_{-9}^{+8}$ & $19_{-1}^{+1}$ \\
\hline $76006 \ldots \ldots \ldots \ldots \ldots$ & $8_{-2}^{+15}$ & $545_{-18}^{+18}$ & $0.69_{-0.33}^{+0.30}$ & $220_{-61}^{+68}$ & $75_{-35}^{+136}$ & $111_{-80}^{+61}$ & $57_{-12}^{+12}$ & $14_{-1}^{+1}$ \\
\hline 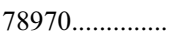 & $13_{-1}^{+7}$ & $871_{-58}^{+76}$ & $0.27_{-0.16}^{+0.53}$ & $663_{-614}^{+183}$ & $53_{-14}^{+178}$ & $100_{-36}^{+66}$ & $61_{-7}^{+10}$ & $22_{-1}^{+2}$ \\
\hline $80884 \ldots \ldots \ldots \ldots . . . . .$. & $14_{-1}^{+2}$ & $1008_{-39}^{+60}$ & $0.46_{-0.13}^{+0.17}$ & $110_{-80}^{+710}$ & $291_{-176}^{+8}$ & $118_{-22}^{+167}$ & $64_{-7}^{+6}$ & $10_{-1}^{+1}$ \\
\hline $84062 \ldots \ldots \ldots \ldots . . . . . .$. & $11_{-1}^{+2}$ & $738_{-46}^{+31}$ & $0.39_{-0.19}^{+0.28}$ & $239_{-188}^{+366}$ & $27_{-10}^{+15}$ & $81_{-48}^{+55}$ & $60_{-9}^{+8}$ & $35_{-1}^{+1}$ \\
\hline $84223 \ldots \ldots \ldots \ldots \ldots$ & $30_{-10}^{+50}$ & $3607_{-1646}^{+5653}$ & $0.73_{-0.32}^{+0.26}$ & $1958_{-1470}^{+5637}$ & $151_{-37}^{+176}$ & $100_{-45}^{+76}$ & $110_{-13}^{+16}$ & $40_{-1}^{+1}$ \\
\hline 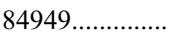 & $15_{-3}^{+9}$ & $1604_{-301}^{+565}$ & $0.76_{-0.17}^{+0.17}$ & $451_{-335}^{+766}$ & $140_{-15}^{+10}$ & $62_{-18}^{+18}$ & $60_{-10}^{+10}$ & $15_{-1}^{+1}$ \\
\hline $85852 \ldots \ldots \ldots \ldots \ldots$ & $8_{-1}^{+1}$ & $903_{-47}^{+48}$ & $0.30_{-0.16}^{+0.23}$ & $640_{-430}^{+178}$ & $181_{-51}^{+155}$ & $111_{-79}^{+99}$ & $135_{-10}^{+12}$ & $11_{-1}^{+1}$ \\
\hline $88848 \ldots \ldots \ldots \ldots \ldots$ & $44_{-8}^{+21}$ & $2053_{-462}^{+995}$ & $0.73_{-0.20}^{+0.10}$ & $660_{-460}^{+845}$ & $253_{-178}^{+4}$ & $103_{-12}^{+173}$ & $78_{-3}^{+3}$ & $30_{-1}^{+1}$ \\
\hline $90355 \ldots \ldots \ldots \ldots . . . . . . .$. & $11_{-2}^{+8}$ & $304_{-11}^{+8}$ & $0.40_{-0.19}^{+0.48}$ & $193_{-58}^{+60}$ & $155_{-33}^{+53}$ & $71_{-47}^{+81}$ & $130_{-19}^{+15}$ & $30_{-2}^{+2}$ \\
\hline $93137 \ldots \ldots \ldots \ldots . . . . .$. & $13_{-2}^{+7}$ & $874_{-52}^{+59}$ & $0.61_{-0.26}^{+0.29}$ & $549_{-134}^{+169}$ & $28_{-5}^{+5}$ & $101_{-39}^{+17}$ & $89_{-5}^{+5}$ & $19_{-1}^{+1}$ \\
\hline $94347 \ldots \ldots \ldots \ldots \ldots$. & $13_{-1}^{+1}$ & $634_{-15}^{+15}$ & $0.24_{-0.11}^{+0.13}$ & $345_{-70}^{+88}$ & $32_{-21}^{+150}$ & $\begin{array}{l}64_{-36}^{+69} \\
\end{array}$ & $146_{-8}^{+10}$ & $23_{-1}^{+1}$ \\
\hline 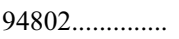 & $18_{-1}^{+1}$ & $1037_{-27}^{+35}$ & $0.11_{-0.06}^{+0.08}$ & $559_{-325}^{+184}$ & $91_{-7}^{+176}$ & $102_{-59}^{+59}$ & $130_{-4}^{+4}$ & $24_{-1}^{+1}$ \\
\hline $97063 \ldots \ldots \ldots \ldots . . .$. & $16_{-1}^{+1}$ & $1376_{-124}^{+427}$ & $0.25_{-0.14}^{+0.36}$ & $848_{-585}^{+435}$ & $48_{-31}^{+175}$ & $\begin{array}{r}-59 \\
96_{-61}^{+63}\end{array}$ & $36_{-10}^{+10}$ & $26_{-1}^{+1}$ \\
\hline $97690 \ldots \ldots \ldots \ldots \ldots$ & $9_{-1}^{+\frac{1}{+2}}$ & $1063_{-60}^{+62}$ & $0.23_{-0.13}^{+0.44}$ & $387_{-289}^{+436}$ & $47_{-16}^{+170}$ & $109_{-55}^{+58}$ & $60_{-9}^{+9}$ & $10_{-1}^{+1}$ \\
\hline 98375................ & $8_{-2}^{+20}$ & $284_{-7}^{+10}$ & $0.77_{-0.40}^{+0.22}$ & $177_{-117}^{+61}$ & $191_{-170}^{+16}$ & $85_{-27}^{+165}$ & $93_{-5}^{+9}$ & $20_{-1}^{+1}$ \\
\hline $103287 \ldots \ldots \ldots \ldots$ & $30_{-2}^{+7}$ & $1347_{-188}^{+573}$ & $0.35_{-0.23}^{+0.38}$ & $873_{-661}^{+406}$ & $339_{-178}^{+5}$ & $127_{-46}^{+161}$ & $88_{-7}^{+6}$ & $25_{-2}^{+2}$ \\
\hline $104440 \ldots \ldots \ldots \ldots$ & $88_{-41}^{+106}$ & $2429_{-1104}^{+3089}$ & $0.65_{-0.07}^{+0.11}$ & $1201_{-784}^{+2500}$ & $17_{-3}^{+180}$ & $147_{-136}^{+32}$ & $91_{-1}^{+1}$ & $51_{-1}^{+1}$ \\
\hline $105969 \ldots \ldots \ldots \ldots$ & $14_{-2}^{+4}$ & $867_{-41}^{+41}$ & $0.42_{-0.22}^{+0.30}$ & $314_{-137}^{+386}$ & $93_{-14}^{+160}$ & $110_{-24}^{+386}$ & $130_{-8}^{+-8}$ & $15_{-2}^{+3}$ \\
\hline $107143 \ldots \ldots \ldots \ldots . . . . . .$. & $35_{-2}^{+5}$ & $1346_{-86}^{+215}$ & $0.29_{-0.16}^{+0.23}$ & $1092_{-940}^{+213}$ & $276_{-175}^{+25}$ & $106_{-63}^{+156}$ & $41_{-10}^{+10}$ & $40_{-2}^{+2}$ \\
\hline $110291 \ldots \ldots \ldots \ldots . . . .$. & $39_{-26}^{+17}$ & $794_{-43}^{+60}$ & $0.99_{-0.19}^{+0.00}$ & $562_{-468}^{+177}$ & $234_{-168}^{+20}$ & $87_{-9}^{+175}$ & $99_{-4}^{+14}$ & $15_{-1}^{+3}$ \\
\hline $113638 \ldots \ldots \ldots \ldots$ & $12_{-3}^{+22}$ & $366_{-9}^{+11}$ & $0.40_{-0.26}^{+0.50}$ & $186_{-77}^{+708}$ & $108_{-89}^{+210}$ & $111_{-44}^{+59}$ & $64_{-12}^{+14}$ & $31_{-8}^{+7}$ \\
\hline $117622 \ldots \ldots \ldots \ldots$ & $13_{-1}^{+3}$ & $1070_{-70}^{+263}$ & $0.44_{-0.17}^{+0.23}$ & $326_{-248}^{+628}$ & $33_{-8}^{+7}$ & $96_{-37}^{+34}$ & $119_{-9}^{+9}$ & $20_{-1}^{+1}$ \\
\hline $118212 \ldots \ldots \ldots \ldots$ & $38_{-5}^{+6}$ & $883_{-28}^{+438}$ & $0.58_{-0.12}^{+0.18}$ & $522_{-90}^{+248}$ & $112_{-5}^{+169}$ & $109_{-23}^{+28}$ & $113_{-5}^{+4}$ & $68_{-3}^{+-1}$ \\
\hline
\end{tabular}


very long period, over 6 yr. It seems that only a small segment of the orbit is present in the Hipparcos data. The parallax is quite stable nevertheless, at 51-52 mas, which is 3-4 mas smaller than the value given in the catalog. Our NDAC-based solution seems the more reasonable for this system, from which a small mass ratio is estimated. The companion to HIP 38910 could be a nearby $\mathrm{M}$ dwarf.

HIP $84223=$ GJ 1213. - This is a nearby $\mathrm{K} 7$ dwarf with an apparently long period. The updated parallaxes are above 40 mas, which warrants keeping this star in the Gliese catalog. With a period of several years and $a_{a}$ probably less than $1 \mathrm{AU}$, the companion could be a late $\mathrm{M}$ dwarf.

HIP $107143=$ GJ 836.3.- This K3 Vp dwarf has a stable solution with $a_{a}=33$ mas and $P \approx 1350$ days. The updated parallax stays just above 40 mas. The companion could be a late $\mathrm{K}$ or early $\mathrm{M}$ dwarf.

HIP $118212=$ GJ 913. - The latest star on our list, this nearby M0 dwarf stands out by virtue of its updated parallax of 68 mas, which is significantly larger than the cataloged value of 58 mas. Our orbital fit is relatively robust, suggesting a late $\mathrm{M}$ dwarf as a companion.

Four early-type stars, HIP 12225 (=HD $16555=\eta$ Hor; A6 V), HIP 32607 (=HD 50241; A7 IV), HIP 39903 (=HD 68456; F5 V), and HIP 62512 (=GJ 9417; F5 V), are prominent X-ray sources detected by ROSAT. Such objects are occasionally found among the general field stars, as well as in nearby open clusters. Since the magnetic activity of the corona is believed to be suppressed in such massive stars because of the vanishing convective zone, it has been suggested that unresolved binary companions of smaller mass are the main contributors to the detected X-ray flux. Finding more astrometric binaries bolsters this surmise. The latter star, HIP 62512 , is of special interest. Beside the strong X-ray radiation, it is also an extreme-UV source and a member of the $\approx 300$ Myr old Ursa Major kinematic group. An astrometric excursion of roughly 1 AU coupled with the $\approx 4$ yr period we determine for this system implies a mass ratio of 0.5 . Thus, the companion could be a young, hot white dwarf. Interestingly, we find three more UMa nucleus members in our sample, HIP 43352 (=HD 75605), HIP 49546, and HIP 61100 (=GJ 1160); the latter is a known spectroscopic binary $(\S 5)$. With these new addition to the list of UMa binaries, the binarity rate in the nucleus of this group becomes uncommonly high.

The star HIP 21965 (=HD 30051), of spectral type F2/F3, is suspected to be very young. Accurate age determination in the upper half of the main sequence is difficult. Our orbital fit suggests a mass ratio of about 0.3 , so that the companion should have a subsolar mass. If resolved, it could yield an accurate isochrone age for the system.

For HIP 23221 (=HD $32008=63$ Eri), we obtain a similar orbit to the previous system, with a slightly longer period of $P \approx$ 850 days. This popular star has in fact been known as a spectroscopic binary, but a poor orbit has been available in the literature. The secondary component is a white dwarf. Our orbital fit suggests a fairly small mass ratio.

\section{SUMMARY}

We present a method for unconstrained optimization of double stars' orbital parameters, based on an adaptation of the genetic evolution algorithm optimization and Monte Carlo simulations for error estimation. This method could become a useful tool for estimating orbital parameters of binary stars and planet hosts for experiments such as SIM, which is expected to observe a limited number of preselected targets. Experiments such as Gaia are expected to observe much higher numbers of objects, and requirements for computing time are likely to become more stringent. However, as the total processing time for the computations in this paper was about 2 weeks of CPU time on a medium-range laptop, we expect the entire Gaia astrometric orbital estimation to become feasible on large-scale scientific clusters in the near future. This "embarrassingly parallel" algorithm is ideally suited for running in a grid environment, and it will take advantage of the ever increasing computer performance. We believe that the simplicity and ease of implementation of our algorithm outweigh the relatively high CPU time requirements.

The 65 stars from the Hipparcos stochastic solution annex are good candidates for follow-up spectroscopic studies, which are expected to yield more accurate orbital parameters constraining the astrometric fits.

\section{Facility: HIPPARCOS}

The research described in this paper was in part carried out at the Jet Propulsion Laboratory, California Institute of Technology, under a contract with the National Aeronautics and Space Administration. This research has made use of the SIMBAD database, operated at CDS, Strasbourg, France.

\section{APPENDIX A}

\section{LINEARIZED EQUATIONS FOR ORBITAL FITS}

The Hipparcos Intermediate Astrometric Data, the basic data set for deriving astrometric orbits, contains partial derivatives of the star abscissae with respect to the five astrometric parameters of the standard model in equatorial coordinates,

$$
d_{1}=\partial a_{i} / \partial \alpha_{*}, \quad d_{2}=\partial a_{i} / \partial \delta, \quad d_{3}=\partial a_{i} / \partial \Pi, \quad d_{4}=\partial a_{i} / \partial \mu_{\alpha *}, \quad d_{5}=\partial a_{i} / \partial \mu_{\delta},
$$

where $a_{i}$ is the abscissa in the $i$ th observation of a given star, $\alpha$ and $\delta$ are the equatorial coordinates, $\alpha_{*}=\alpha \cos \delta$, $\Pi$ is the parallax, and $\mu_{\alpha *}=\mu_{\alpha} \cos \delta$ and $\mu_{\delta}$ are the orthogonal proper-motion components. The abscissa is defined as a great-circle arc connecting an arbitrarily chosen reference zero point and the star on a fixed great circle (close to the scan circle).

In the small-angle approximation, a linearized equation for the observed abscissa difference $\Delta a_{i}=a_{\text {obs }}-a_{\text {calc }}$ can be written as

$$
d_{1} \Delta x+d_{2} \Delta y+d_{3} \Delta \Pi+d_{4} \Delta \mu_{x}+d_{5} \Delta \mu_{y}+d_{1} \sum_{j} \frac{\partial x}{\partial \epsilon_{j}} \Delta \epsilon_{j}+d_{2} \sum_{j} \frac{\partial y}{\partial \epsilon_{j}} \Delta \epsilon_{j}=\Delta a_{i},
$$

where $\epsilon_{j}$ are the elements of the vector of seven orbital elements, $\epsilon=\left(a_{a}, P, e, T_{0}, \omega, \Omega, i\right)$. As usual, $a_{a}$ is the semimajor axis of the apparent orbital motion (i.e., the motion of the photocenter), $P$ is the orbital period in years, $e$ is the eccentricity, $T_{0}$ is the periastron time, 
$\omega$ is the longitude of the ascending node in the orbit plane in radians, $\Omega$ is the position angle of the node in the plane of projection, and $i$ is the inclination of the orbit (zero for face-on orbits). In this equation, the notations $\alpha_{*}$ and $\delta$ have been changed to $x$ and $y$, respectively, to make them consistent with the traditionally used tangential coordinates for apparent orbits. Equation (A2) holds only in the vicinity of a certain point in the twelve-dimensional parameter space $\left\{\alpha, \delta, \Pi, \mu_{\alpha *}, \mu_{\delta}, \epsilon\right\}$, as long as the corrections to these parameters remain small.

The apparent motion of a binary in the plane of celestial projection is described by

$$
x=A(\cos E-e)+F \sqrt{1-e^{2}} \sin E, \quad y=B(\cos E-e)+G \sqrt{1-e^{2}} \sin E
$$

(Heintz 1978), where $x$ and $y$ are the tangential coordinates and $E$ is the eccentric anomaly, related to the mean anomaly $M$ by Kepler's equation

$$
M=2 \pi \frac{T-T_{0}}{P}=E-e \sin E .
$$

The Thiele-Innes constants are related to the remaining orbital elements by

$$
\begin{aligned}
A=a_{a}(\cos \omega \cos \Omega-\sin \omega \sin \Omega \cos i), & & B=a_{a}(\cos \omega \sin \Omega+\sin \omega \cos \Omega \cos i), \\
F=a_{a}(-\sin \omega \cos \Omega-\cos \omega \sin \Omega \cos i), & & G=a_{a}(-\sin \omega \sin \Omega+\cos \omega \cos \Omega \cos i),
\end{aligned}
$$

where $a_{a}$ is the apparent semimajor axis of the photocenter, $\omega$ is the periastron longitude, $\Omega$ is the node, and $i$ is the orbit inclination $\left(i=90^{\circ}\right.$ is an edge-on orbit).

\section{APPENDIX B}

\section{COVARIANCES WITH LINEARIZED EQUATIONS}

Explicitly, the partial derivatives $\partial x / \partial \epsilon_{j}$ and $\partial y / \partial \epsilon_{j}$ in equation (A2) are

$$
\begin{aligned}
& \frac{\partial x}{\partial a_{a}}=\frac{x}{a_{a}}, \quad \frac{\partial y}{\partial a_{a}}=\frac{y}{a_{a}}, \quad \frac{\partial x}{\partial P}=\left(-A \sin E+F \sqrt{1-e^{2}} \cos E\right) \frac{\partial E}{\partial P}, \quad \frac{\partial y}{\partial P}=\left(-B \sin E+G \sqrt{1-e^{2}} \cos E\right) \frac{\partial E}{\partial P}, \\
& \frac{\partial x}{\partial e}=-A\left(1+\sin E \frac{\partial E}{\partial e}\right)+F \frac{\sin E(\cos E-e)}{\sqrt{1-e^{2}}(1-e \cos E)}, \quad \frac{\partial y}{\partial e}=-B\left(1+\sin E \frac{\partial E}{\partial e}\right)+G \frac{\sin E(\cos E-e)}{\sqrt{1-e^{2}}(1-e \cos E)}, \\
& \frac{\partial x}{\partial T_{0}}=\left(-A \sin E+F \sqrt{1-e^{2}} \cos E\right) \frac{\partial E}{\partial T_{0}}, \quad \frac{\partial y}{\partial T_{0}}=\left(-B \sin E+G \sqrt{1-e^{2}} \cos E\right) \frac{\partial E}{\partial T_{0}}, \\
& \frac{\partial x}{\partial \omega}=(\cos E-e) \frac{\partial A}{\partial \omega}+\sqrt{1-e^{2}} \sin E \frac{\partial F}{\partial \omega}, \quad \frac{\partial y}{\partial \omega}=(\cos E-e) \frac{\partial B}{\partial \omega}+\sqrt{1-e^{2}} \sin E \frac{\partial G}{\partial \omega}, \\
& \frac{\partial x}{\partial \Omega}=(\cos E-e) \frac{\partial A}{\partial \Omega}+\sqrt{1-e^{2}} \sin E \frac{\partial F}{\partial \Omega}, \quad \frac{\partial y}{\partial \Omega}=(\cos E-e) \frac{\partial B}{\partial \Omega}+\sqrt{1-e^{2}} \sin E \frac{\partial G}{\partial \Omega}, \\
& \frac{\partial x}{\partial i}=(\cos E-e) \frac{\partial A}{\partial i}+\sqrt{1-e^{2}} \sin E \frac{\partial F}{\partial i}, \quad \frac{\partial y}{\partial i}=(\cos E-e) \frac{\partial B}{\partial i}+\sqrt{1-e^{2}} \sin E \frac{\partial G}{\partial i},
\end{aligned}
$$

where

$$
\begin{gathered}
\frac{\partial E}{\partial P}=\frac{2 \pi\left(T-T_{0}\right)}{P^{2}(e \cos E-1)}, \quad \frac{\partial E}{\partial e}=\frac{\sin E}{1-e \cos E}, \quad \frac{\partial E}{\partial T_{0}}=\frac{-2 \pi}{P(1-e \cos E)}, \\
\frac{\partial A}{\partial \omega}=F, \quad \frac{\partial B}{\partial \omega}=G, \quad \frac{\partial F}{\partial \omega}=-A, \quad \frac{\partial G}{\partial \omega}=-B, \quad \frac{\partial A}{\partial \Omega}=-B, \quad \frac{\partial B}{\partial \Omega}=A, \quad \frac{\partial F}{\partial \Omega}=-G, \quad \frac{\partial G}{\partial \Omega}=F, \\
\frac{\partial A}{\partial i}=a_{a} \sin \omega \sin \Omega \sin i, \quad \frac{\partial B}{\partial \omega}=-a_{a} \sin \omega \cos \Omega \sin i, \quad \frac{\partial F}{\partial \omega}=a_{a} \cos \omega \sin \Omega \sin i, \quad \frac{\partial G}{\partial \omega}=-a_{a} \cos \omega \cos \Omega \sin i .
\end{gathered}
$$

Using these relations, the design matrix $D$ can be computed from equation (A2), and the covariance matrix $C=\left(D^{T} D\right)^{-1}$ is readily computed. Astrometric abscissae $a_{i}$ for the same star may have different standard errors in the HIAD, making it necessary to employ a weighted least-squares routine.

Abt, H. A., \& Levy, S. G. 1985, ApJS, 59, 229

Andrievsky, S. M., \& Paunzen, S. 2000, MNRAS, 313, 547

Carrier, F., North, P., Udry, S., \& Babel, J. 2002, A\&A, 394, 151

de Medeiros, J. R., Da Silva, J. R. P., \& Maia, M. R. G. 2002, ApJ, 578, 943

de Medeiros, J. R., \& Mayor, M. 1999, A\&AS, 139, 433

ESA. 1997, The Hipparcos and Tycho Catalogues, Vol. 3 (ESA SP-1200)

(Noordwijk: ESA)

\section{REFERENCES}

Fabricius, C., \& Makarov, V. V. 2000, A\&AS, 144, 45

Falin, J.-L., \& Mignard, F. 1999, A\&AS, 135, 231

Faraggiana, R., \& Bonifacio, P. 2005, A\&A, 436, 697

Faraggiana, R., Bonifacio, P., Caffau, E., Gerbaldi, M., \& Nonino, M. 2004, A\&A, 425, 615

Fekel, F. C., Barlow, D. J., Scarfe, C. D., Jancart, S., \& Pourbaix, D. 2005, AJ, 129,1001 
Fekel, F. C., Henry, G. W., Busby, M. R., \& Eitter, J. J. 1993, AJ, 106, 2370 Freire Ferrero, R., Frasca, A., Marilli, E., \& Catalano, S. 2004, A\&A, 413, 657

Grenier, S., Burnage, R., Faraggiana, R., Gerbaldi, M., Delmas, F., Gómez, A. E., Sabas, V., \& Sharif, L. 1999, A\&AS, 135, 503

Halbwachs, J.-L., Arenou, F., Mayor, M., Udry, S., \& Queloz, D. 2000, A\&A, 355,581

Halbwachs, J.-L., Mayor, M., Udry, S., \& Arenou, F. 2003, A\&A, 397, 159

Hastie, T., Tibshirani, R., \& Friedman, J. 2001, The Elements of Statistical Learning (New York: Springer)

Heintz, W. D. 1978, Double Stars (Dordrecht: Reidel)

Latham, D. W., Stefanik, R. P., Torres, G., Davis, R. J., Mazeh, T., Carney, B. W., Laird, J. B., \& Morse, J. A. 2002, AJ, 124, 1144
Makarov, V. V., \& Kaplan, G. H. 2005, AJ, 129, 2420

Nordström, B., et al. 2004, A\&A, 418, 989

Pourbaix, D., \& Jorissen, A. 2000, A\&AS, 145, 161

Scarfe, C. D., Barlow, D. J., Fekel, F. C., Rees, R. F., Lyons, R. W., Bolton, C. T., McAlister, H. A., \& Hartkopf, W. I. 1994, AJ, 107, 1529

Setiawan, J., Pasquini, L., da Silva, L., Hatzes, A. P., von der Lühe, O., Girardi, L., de Medeiros, J. R., \& Guenther, E. 2004, A\&A, 421, 241

Storn, R., \& Price, K. 1995, Differential Evolution: A Simple and Efficient Adaptive Scheme for Global Optimization over Continuous Spaces (ICSI Tech. Rep. TR-95-012) (Berkeley: Int. Comput. Sci. Inst.)

Torres, G. 2006, AJ, 131, 1022

Van Hamme, W. V., et al. 1994, AJ, 107, 1521 